\title{
Stochastic Simulation of Graph Transformation Systems
}

\author{
Paolo Torrini ${ }^{1}$, Reiko Heckel ${ }^{1}$, and István Ráth ${ }^{2}$ \\ 1 Department of Computer Science, University of Leicester \\ pt95, reiko@mcs.le.ac.uk \\ 2 Department of Measurement and Information Systems \\ Budapest University of Technology and Economics \\ rath@mit.bme.hu
}

\section{Introduction}

Stochastic graph transformation systems (SGTS) [1] support integrated modelling of architectural reconfiguration and non-functional aspects such as performance and reliability. In its simplest form a SGTS is a graph transformation system (GTS) where each rule name is associated with a rate of an exponential distribution governing the delay of its application. However, this approach has its limitations. Model checking with explicit states does not scale well to models with large state space. Since performance and reliability properties often depend on the behaviour of large populations of entities (network nodes, processes, services, etc.), this limitation is significant. Also, exponential distributions do not always provide the best abstraction. For example, the time it takes to make a phone call or transmit a message is more likely to follow a normal distribution.

To counter these limitations, generalised SGTS [2] allow for general distributions dependent on rule - match pairs (rather than just rule names). Generalised semi-Markov processes provide a semantic model for such systems, supporting stochastic simulation. Rather than model checking, simulations provide a more flexible tradeoff between analysis effort and confidence in the result and so allow to verify soft performance targets in large-scale systems.

We present a tool called GraSS, for Graph-based Stochastic Simulation, to enable the analysis of such processes. The tool is developed in Java-Eclipse, extending the VIATRA model transformation plugin with a control based on the SSJ library for Stochastic Simulation in Java. The main performance challenge, in finding, at each state of the simulation, all matches for all rules, is alleviated by VIATRA's RETE-style incremental pattern-matching approach [3], which stores precomputed matching information and updates it during transformation. We illustrate and evaluate the application of the tool by the simulation of the original $\mathrm{P} 2 \mathrm{P}$ reconfiguration model as well as an improved and scaled-up version.

\section{A P2P Network Model}

As a test case we use an example of a SGTS modelling reconfigurations in a P2P network [1]. Generating the state space of the model for up to seven peers, 
in [1] we used stochastic model checking to analyse, e.g., the probability of the network being fully connected, so that each participant can communicate with every other one.

The GTS below models basic P2P network reconfigurations. Rule new on the left adds a new peer, registers it and links it to an existing peer. Rule kill deletes a peer with all links attached. Predicate disconnected checks if there are two nodes that are not connected by a path of links labelled $l$.

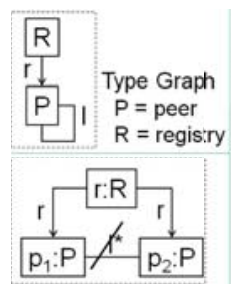

disconnected

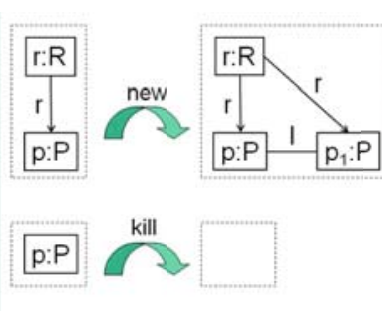

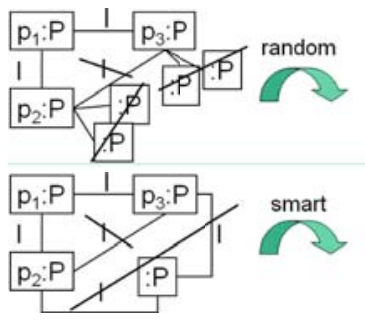

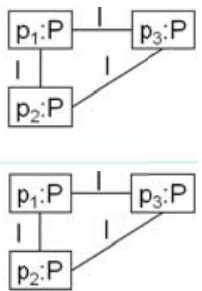

The two rules on the right create redundant links to increase reliability in case a peer is lost. Rule random creates a link between $p 2$ and $p 3$ unless there is one already or the number of additional connections of either $p 2$ or $p 3$ is greater than two. Rule smart creates a link if there is no two-hop path between $p 2$ and $p 3$ apart from the one via $p 1$. We consider two families of systems, $S G T S_{\text {random,x }}$ and $S G T S_{\text {smart }, x}$. The former has rules $\{$ new, kill, random $\}$ and rates $\sigma($ new $)=\sigma($ kill $)=1$ and $\sigma$ (random $)=x$. In the latter, random is replaced by smart with $\sigma($ smart $)=x$. In both cases $x$ ranges from 1 to 10,000 to test different ratios between basic and redundancy rules.

\section{Simulating Stochastic Graph Transformations}

In order to define a general interface between the stochastic control component of the simulation and existing graph transformation tools used for executing rules, we define SGTS for a generic notion of graph transformation. Refining [4, a graph transformation approach is given by a class of graphs $\mathcal{G}$, a class of rules $\mathcal{R}$, and a $\mathcal{R} \times \mathcal{G}$-indexed family of sets of rule matches $\mathcal{M}_{r, G}$ for rule $r$ into graph $G$. Transformation is defined by a family of partial functions $\Rightarrow_{r, m}: \mathcal{G} \rightarrow \mathcal{G}$, such that $\Rightarrow_{r, m}(G)$ is defined if and only if $m \in \mathcal{M}_{r, G}$. This captures the idea that rule application is well-defined and deterministic if $m$ is a match for $r$ in $G$.

For a set of rules $R, \mathcal{E}_{R}$ is the set of events, i.e., compatible pairs $\langle r, m\rangle$. $\mathcal{S}=\left\langle R, G_{0}, F\right\rangle$ is a stochastic graph transformation system with set of rules $R$, initial graph $G_{0}$, and $F: \mathcal{E}_{R} \rightarrow(\mathbf{R} \rightarrow[0,1])$ assigning each event a continuous distribution function such that $F(e)(0)=0$.

We encode SGTS into generalised semi-Markov schemes (GSMS), a generalisation of Markov chains associated with generalised semi-Markov processes [5]. Here transitions are independent of past states, but unlike Markov chains they may depend on the time spent in the current one, i.e., interevent times may be non-exponentially distributed. Formally, a GSMS is a structure

$\mathcal{P}=\langle S, E$, act $: S \rightarrow \wp(E)$, trans $: S \times E \rightarrow S, \delta: E \rightarrow(\mathcal{R} \rightarrow[0,1])$, init $: S\rangle$ 
where $S$ is a set of states (given by all graphs reachable in $\mathcal{S}$ ), $E$ is a set of events (the rule matches $\mathcal{E}_{R}$ ), init is the initial state (graph $G_{0}$ ), act gives the set of events (rule matches) enabled in a state (graph), trans is the transition function (given by $\operatorname{trans}(G,\langle r, m\rangle)=\Rightarrow_{r, m}(G)$ ), and $\delta$ defines the probability distribution for each event (given by $F$ ).

The simulation component uses VIATRA as a graph transformation tool to implement the elements of the GSMS that depend on the representation of states and events, notably $S, E$, act, trans, init, i.e., GTSs are represented as a VIATRA models. Definitions of distributions $F$ are loaded from an XML file. Based on this data, a GSMS simulation in GraSS consists of the following steps

1. Initialisation - the simulation time $T$ is initialised to 0 and the set of the enabled matches (active events) is obtained from the graph transformation engine. For each active event, a scheduling time $t_{e}$ is computed by a random number generator (RNG) based on the probability distribution assigned to the event. Timed events are collected as a list ordered by time (state list).

2. At each simulation step

(a) the first element $k=(e, t)$ is removed from the state list

(b) the simulation time is increased to $t$

(c) the event $e$ is executed by the graph transformation engine

(d) the new state list $s^{\prime}$ is computed, by querying the engine, removing all the elements that have been disabled, adding to the list an event for each newly enabled match $m$ with time $t=T+d$, where $d$ is provided by the RNG depending on $F(m)$, and reordering the list with respect to time

GT rules with empty postconditions are used as probes — statistics about occurrence of precodition patterns are computed as SSJ tally class reports, giving average values over runs. One can specify the number of runs per experiment (esp. useful to reduce the biasing effect of runs truncated by deletion of all elements) and their max depth (either by number of steps or simulation time).

\section{Evaluation}

In order to validate the correctness and scalability of the tool we run a number of experiments based on the P2P model of Section 2. We do not expect to replicate exactly the results reported in [1] because (1) we remove the restriction to 7 nodes that was used to guarantee a finite (and manageable) state space; (2) unlike in [1] where states and transitions were presented up to isomorphism, our simulation deals with concrete graphs and transitions. A detailed comparison of the underlying mathematical models is beyond the scope of this paper, but it appears that, since the Markov chain is constructed from a more abstract transition system in [1], the two are not in stochastic bisimulation. Thus, evaluating the same properties on both models may lead to different results. As in [1] we run experiments with 10 different models, 5 versions each of using random and smart rules, with rates ranging through $x \in\{1,10,100,1000,10000\}$. 
We perform 5 runs each with a simulation time bound of 10 s for each experiment - i.e. no run exceeds 10 s regardless of the number of steps. The table below gives the output of an experiment, indicating the version of the model (1st column) followed by the percentage of disconnected states encountered, the average number of steps performed per run, the average maximal extension of the network, and the average time taken for each run.

\begin{tabular}{lcccc}
\hline \hline Model: P2P & Disconnected Number of steps Max number of peers Runtime \\
\hline random:1 & 0.46 & 33 & 6 & 5 \\
random:10 & 0.62 & 71 & 8 & 8 \\
random:100 & 0.55 & 86 & 8 & 7 \\
random:1000 & 0.89 & 284 & 20 & 10 \\
random:10,000 & 0.46 & 116 & 8 & 9 \\
smart:1 & 1.33 & 18 & 5 & 1 \\
smart:10 & 0.01 & 90 & 8 & 4 \\
smart:100 & 0.00 & 3561 & 48 & 10 \\
smart:1000 & 0.00 & 998 & 24 & 10 \\
smart:10,000 & 0.00 & 62 & 8 & 3 \\
\hline
\end{tabular}

Such results confirm the inverse dependency observed in [1] between the rate of the smart rule and the probability of being disconnected, whereas for the random rule an increased rate does not lead to any significant change in reliability - as confirmed by the average number of disconnections modulo square of node number (not shown). The performance (number of simulation steps per sec) is limited by the complexity of pattern disconnect which, in a network of $n$ peers, checks for (non-) existence of $n^{2}$ paths. This can be hard due to transitive closure. As a simpler reliability measure, the proportion of peers with at least two connections (hence less vulnerable to loss of connectivity) can do. A simulation of 5 runs with a time limit of 10 s has always been carried out in less than a minute. Reliance on incremental pattern matching means model size only affects simulation up to number of RNG calls, whereas increase in number and complexity of the rules can add to the cost of graph transformation, too.

\section{References}

1. Heckel, R.: Stochastic analysis of graph transformation systems: A case study in P2P networks. In: Van Hung, D., Wirsing, M. (eds.) ICTAC 2005. LNCS, vol. 3722, pp. 53-69. Springer, Heidelberg (2005)

2. Khan, A., Torrini, P., Heckel, R.: Model-based simulation of VoIP network reconfigurations using graph transformation systems. In: Corradini, A., Tuosto, E. (eds.) ICGT 2008. El. Com. EASST, vol. 16, pp. 1-20 (2008)

3. Bergmann, G., Ökrös, A., Ráth, I., Varró, D., Varró, G.: Incremental pattern matching in the Viatra model transformation system. In: GRaMoT 2008, pp. 25-32. ACM, New York (2008)

4. Kreowski, H.J., Kuske, S.: On the interleaving semantics of transformation units - a step into GRACE. In: Cuny, J., Engels, G., Ehrig, H., Rozenberg, G. (eds.) Graph Grammars 1994. LNCS, vol. 1073, pp. 89-106. Springer, Heidelberg (1996)

5. D'Argenio, P.R., Katoen, J.P.: A theory of stochastic systems part I: Stochastic automata. Inf. Comput. 203(1), 1-38 (2005) 